Fortschritte der

Neurologie - Psychiatrie

Thieme

\title{
Tragbare und aktiv vom Parkinson-Patienten genutzte Technologie im häuslichen Umfeld: Was bringt die Zukunft?
}

\begin{tabular}{|c|c|}
\hline Journal: & Fortschritte der Neurologie - Psychiatrie \\
\hline Manuscript ID & FDNP-0073-01-2016-SU \\
\hline Manuscript Type: & Supplement \\
\hline Date Submitted by the Author: & 12-Jan-2016 \\
\hline Complete List of Authors: & $\begin{array}{l}\text { Maetzler, Walter; Universitätsklinikum Tübingen, Neurologie mit } \\
\text { Schwerpunkt Neurodegenerative Erkrankungen, Hertie Institut für klin. } \\
\text { Hirnforschung; DZNE, Deutsches Zentrum für Neurodegenerative } \\
\text { Erkrankungen } \\
\text { Krüger, Rejko; Universitätsklinikum Tübingen, Neurologie mit Schwerpunkt } \\
\text { Neurodegenerative Erkrankungen, Hertie Institut für klin. Hirnforschung; } \\
\text { DZNE, Deutsches Zentrum für Neurodegenerative Erkrankungen; } \\
\text { University of Luxembourg, and Centre Hospitalier de Luxembourg, Clinical } \\
\text { and Experimental Neuroscience, Luxembourg Centre for Systems } \\
\text { Biomedicine } \\
\text { Mueller, Thomas; St Joseph Krankenhaus Berlin Weissensee, Klinik für } \\
\text { Neurologie } \\
\text { Oertel, Wolfgang; Universitätsklinikum Gießen und Marburg GmbH, } \\
\text { Standort Marburg, Klinik für Neurologie } \\
\text { Urban, Peter; Asklepios Klinik Barmbek, Abteilung für Neurologie } \\
\text { Warnecke, Tobias; Universitatsklinikum Munster, Neurologie } \\
\text { Klucken, Jochen; Universitätsklinikum Erlangen, Molekulare Neurologie }\end{array}$ \\
\hline Keywords: & Häusliches Umfeld, quantitatives Assessment, Sensoren \\
\hline \multirow[t]{2}{*}{ Keywords (2nd language): } & Body-worn sensors, continuous evaluation, quantiative assessment \\
\hline & $\begin{array}{l}\text { Das Idiopathische Parkinson-Symptom weist eine Vielzahl von Symptomen } \\
\text { auf, deren Anzahl und Ausprägung weit über den üblicherweise in der Klinik } \\
\text { oder Facharztpraxis erfassten Bereich hinausgehen, zum Teil auch nur im } \\
\text { häuslichen Umfeld auftreten und somit der Untersuchung durch den Arzt } \\
\text { entgehen. Moderne Entwicklungen im Bereich der tragbaren } \\
\text { Bewegungssensorik könnten hier Abhilfe schaffen. Im Gesundheits- und } \\
\text { Fitnessbereich finden diese neuen Technologien durch kleine, tragbare und } \\
\text { vom Nutzer selbst bedienbare Sensoren bereits erste Anwendungen. Da die } \\
\text { klinische Erfassung von Krankheitssymptomen verschiedensten } \\
\text { Limitationen unterliegen (subjektiv, ungenau, Momentaufnahme einer im } \\
\text { Verlauf stark schwankenden Krankheit, Zeitaufwand), liegt es nahe, über } \\
\text { Sensoren Bewegungseinschränkungen von Parkinsonpatienten auch im } \\
\text { häuslichen Umfeld zu erfassen. Erste Studien zur Erfassung von } \\
\text { Bradykinese, Dyskinesie, Tremor, Tagesaktivität und Bewegungen im } \\
\text { Schlaf deuten darauf hin, dass derartige Sensortechnik eine sinnvolle } \\
\text { Ergänzung der klinischen Diagnostik darstellt, und in naher Zukunft in die }\end{array}$ \\
\hline
\end{tabular}


Routineversorgung von Parkinsonpatienten eingebunden werden kann. Die aktuelle Entwicklung hat darüber hinaus das Potential, Patienten in Zukunft aktiv in die Diagnostik und Therapie-Evaluation einzubinden.

Parkinson's disease (PD) is a multisystem disorder with a plethora of symptoms affecting quality of life in the home environment. Due to the rapid development of wearable technique in the health and fitness sector, an increasing number of such wearables are available to complement diagnostic strategies of PD symptoms not only in the clinical but also in the domestic environment. This development has clear advantages over clinical evaluation, as the latter is relatively subjective, time-consuming and costly, and provides only a snapshot of the condition. First results about the use of such technology for the assessment of PD symptoms (including bradykinesia, dyskinesia, tremor, daily activity and sleep behavior) in the domestic environment are promising. They suggest that these techniques can provide complementary information about the symptoms of PD patients, and have the potential to be included in future diagnostic workup concepts of routine care in PD. The use of such technique provides also the chance to more actively include patients into medical decision making processes. 
Tragbare und aktiv vom Parkinson-Patienten genutzte Technologie im häuslichen Umfeld: Was bringt die Zukunft?

Walter Maetzler ${ }^{1,2}$, Rejko Krüger ${ }^{1,2,3}$, Thomas Müller ${ }^{4}$, Wolfgang Oertel ${ }^{5}$, Peter Urban ${ }^{6}$, Tobias Warnecke ${ }^{7}$, Jochen Klucken ${ }^{8}$

${ }^{1}$ Neurologie mit Schwerpunkt Neurodegeneration und Hertie Institut für klinische Hirnforschung, Universitätsklinikum Tübingen, Tübingen

${ }^{2}$ Deutsches Zentrum für Neurodegenerative Erkrankungen (DZNE), Tübingen

${ }^{3}$ Clinical and Experimental Neuroscience, Luxembourg Centre for Systems Biomedicine, University of Luxembourg, and Centre Hospitalier de Luxembourg, Luxembourg

${ }^{4}$ Klinik für Neurologie, St Joseph Krankenhaus Berlin Weissensee, Berlin

${ }^{5}$ Klinik für Neurologie, Universitätsklinikum Gießen und Marburg GmbH, Standort Marburg, Marburg

${ }^{6}$ Abteilung für Neurologie, Asklepios Klinik Barmbek, Hamburg

${ }^{7}$ Department für Neurologie, Universitätsklinikum Münster, Münster

${ }^{8}$ Molekulare Neurologie, Universitätsklinikum Erlangen, Friedreich-Alexander Universität ErlangenNürnberg (FAU), Erlangen

Korrespondenz: Prof. Walter Maetzler, Neurologische Universitätsklinik, Hoppe-Seyler-Strasse 3, 72076 Tübingen, Tel 07071 29-82141, Fax 07071 29-25087, walter.maetzler@uni-tuebingen.de

Interessenskonflikte: Die Autoren geben keine Interessenskonflikte an. 


\section{Zusammenfassung}

Das Idiopathische Parkinson-Symptom weist eine Vielzahl von Symptomen auf, deren Anzahl und Ausprägung weit über den üblicherweise in der Klinik oder Facharztpraxis erfassten Bereich hinausgehen, zum Teil auch nur im häuslichen Umfeld auftreten und somit der Untersuchung durch den Arzt entgehen. Moderne Entwicklungen im Bereich der tragbaren Bewegungssensorik könnten hier Abhilfe schaffen. Im Gesundheits- und Fitnessbereich finden diese neuen Technologien durch kleine, tragbare und vom Nutzer selbst bedienbare Sensoren bereits erste Anwendungen. Da die klinische Erfassung von Krankheitssymptomen verschiedensten Limitationen unterliegen (subjektiv, ungenau, Momentaufnahme einer im Verlauf stark schwankenden Krankheit, Zeitaufwand), liegt es nahe, über Sensoren Bewegungseinschränkungen von Parkinsonpatienten auch im häuslichen Umfeld zu erfassen. Erste Studien zur Erfassung von Bradykinese, Dyskinesie, Tremor, Tagesaktivität und Bewegungen im Schlaf deuten darauf hin, dass derartige Sensortechnik eine sinnvolle Ergänzung der klinischen Diagnostik darstellt, und in naher Zukunft in die Routineversorgung von Parkinsonpatienten eingebunden werden kann. Die aktuelle Entwicklung hat darüber hinaus das Potential, Patienten in Zukunft aktiv in die Diagnostik und Therapie-Evaluation einzubinden.

\section{Abstract}

Parkinson's disease (PD) is a multisystem disorder with a plethora of symptoms affecting quality of life in the home environment. Due to the rapid development of wearable technique in the health and fitness sector, an increasing number of such wearables are available to complement diagnostic strategies of PD symptoms not only in the clinical but also in the domestic environment. This development has clear advantages over clinical evaluation, as the latter is relatively subjective, timeconsuming and costly, and provides only a snapshot of the condition. First results about the use of such technology for the assessment of PD symptoms (including bradykinesia, dyskinesia, tremor, daily activity and sleep behavior) in the domestic environment are promising. They suggest that these techniques can provide complementary information about the symptoms of PD patients, and have the potential to be included in future diagnostic workup concepts of routine care in PD. The use of such technique provides also the chance to more actively include patients into medical decision making processes. 
Das Idiopathische Parkinson-Syndrom (IPS) ist eine chronisch fortschreitende neurologische Erkrankung, bei der neben motorischen Symptomen zahlreiche nichtmotorische Symptome, wie kognitive Einschränkungen, Halluzinationen und autonome Dysfunktion vorliegen können. Der jeweilige klinische Befund wird vom betreuenden Arzt in regelmäßigen Abständen durch Anamneseerhebung und klinische Evaluation erhoben. Bei der klinischen Evaluation können validierte Skalen zur Beurteilung der bestehenden Symptome herangezogen werden. Häufig verwendete Skalen sind die Unified Parkinson's Disease Rating Scale (UPDRS), die Hoehn\&Yahr-Skala und für kognitive Screening-Untersuchungen der Mini-Mental-Test (MMSE) oder das Montreal Cognitive Assessment (MoCA). Derartige Skalen haben den Vorteil, dass Defizite zumindest semiquantitativ erfasst werden können. Sie haben aber auch Nachteile, wie Untersucherabhängigkeit, hoher Zeit- und Personalaufwand, Erfassung nur einer Momentaufnahme, Lokalisation der Erhebung in einem für den Patienten unüblichen Rahmen (und damit Möglichkeit der Verfälschung der Ergebnisse), Erhebung von im Alltag nicht relevanten Symptomen und umgekehrt Nichterhebung von Alltags-relevanten Symptomen ${ }^{1}$. Die Nachteile dieser Skalen wirken sich möglicherweise auch auf die Entwicklung neuer Therapien aus: derartige Skalen werden als Endpunkte von Therapiestudien verwendet, was den Nachteil beinhaltet, dass Therapie-relevante Veränderungen im Verlauf der Erkrankung übersehen werden können bzw. sehr große Gruppengrößen für die Testung der Wirksamkeit von Therapien verwendet werden müssen. All diese Aspekte machen klar, dass im Bereich des IPS ein großer Bedarf an neuen Erfassungsmöglichkeiten insbesondere für den Verlauf der Erkrankung und für die Evaluierung des Therapie-Erfolgs besteht.

Im Verlauf des letzten Jahrzehntes zeigte sich im Gesundheits- und Fitnessbereich eine hochdynamische Entwicklung bezüglich tragbarer Sensoren bzw. Sensortechnik im „üblichen“ Smartphone, wie auch im Bereich Datenübertragung und Datensicherheit im Netz. Es findet sich heute in praktisch jedem größeren Elektronikgeschäft eine Abteilung, in der solche Sensorsysteme dem Konsumenten für den täglichen Gebrauch angeboten werden. Derartige Sensorsysteme eignen sich allerdings nicht nur für die Erfassung von Fitness- und Gesundheitsdaten unter der Voraussetzung von motorischer Gesundheit im engen Sinn, sondern können prinzipiell auch für die Erfassung von Krankheitssymptomen verwendet werden ${ }^{2,3}$. Dies hat in der Wissenschaft dazu geführt, vielversprechende Sensoren, Sensorsysteme und Algorithmen zu entwickeln, welche IPSrelevante Symptome messen können. Die Attraktivität derartiger Systeme und Algorithmen wird dadurch erhöht, dass die Datenerhebung nun nicht mehr im Labor, in der Praxis oder Klinik erfolgt, sondern grundsätzlich und auch vergleichend im häuslichen Umfeld möglich ist. Symptome, welche 
nach derzeitiger Studienlage relativ gut mit derartigen Systemen und Algorithmen erfasst werden können, sind Tremor, Bewegungen während des Schlafs, Bradykinese, Dyskinesie, Gangstörungen und (Einschränkungen von) Stand- und Sitztransfer ${ }^{4}$. Ein Nachteil dieser Systeme ist, dass sie präferentiell Motorik erfassen, und bekannt ist, dass Motorik im erheblichen Maß auch durch Emotion beeinflusst wird. Vorteil ist, dass Motorik über einen längeren Zeitraum und nicht während eines bestimmten Zeitpunktes erfasst wird (z.B. Diadochokineseprüfung ${ }^{5}$ ).

\section{Bradykinese}

Die Erfassung der Bradykinese (besser Hypokinese) erfolgt zumindest in einigen von den derzeit vorliegenden Studien durch Tragen von Sensoren, meist über mehrere Tage, z.B. am Arm (wie eine Armbanduhr) oder im Bereich des Gürtels oder des unteren Rückenbereichs. Als eine mögliche Art der Darstellung zerlegt ein Algorithmus die aufgezeichnete Zeit in Intervalle von wenigen Minuten, welche dann jeweils hinsichtlich Vorkommen von abnormen Bewegungsmustern, und Beurteilung des Ausmaßes von Bewegung klassifiziert werden ${ }^{6}$. Daraus lassen sich Muster von verminderter Bewegung über längere Zeitverläufe erstellen, welche wiederum indirekt Rückschlüsse auf Effektivität der Therapie (z.B. Medikamentenwirkung, Stimulator-Einstellungen bei Tiefer Hirnstimulation) oder Aktivität zulassen können.

\section{Dyskinesie}

Die Erfassung der Dyskinesie erfolgt in einzelnen Studien wie oben berichtet für Hypokinese, nur dass nun kurze Intervalle, in denen viel Bewegung und besonders viel Bewegungsaktivität an den Positionen in denen Sensoren befestigt waren, stattgefunden hat ${ }^{6}$. Ein anderer Ansatz ist die Quantifizierung von Bewegung durch Sensoren, welche nichtphysiologische Bewegungsmuster aufzeichnen (z.B. Dyskinesien im Bereich der Beine haben vermehrt mediolaterale und rotatorische Komponenten, während physiologische Bewegungen vor allem antero-posterior ausgerichtet sind).

\section{Gang}

Es wurde wiederholt gezeigt, dass IPS-Patienten (vor allem unter Multitasking-Situationen) dazu neigen, eine erhöhte Gangvariabilität zu haben. Dies ist mit dem Risiko von (gehäuften) Stürzen assoziiert. Mit Daten von tragbaren Sensorsystemen ist es mehrfach gelungen, nicht nur die Schrittanzahl über einen bestimmten Zeitraum zu detektieren, sondern auch Parameter aus den 
Daten zu extrahieren, welche Aussagen über Gangvariabilität ermöglichen. Aus dem Parkinsontypischen Gangmuster sind auch diagnostische Klassifikationen des Stadiums und der motorischen Einschränkung der Patienten möglich ${ }^{7}$. Des Weiteren ist es gelungen, auch Drehbewegungen zu beurteilen ${ }^{8}$. Drehbewegungen spielen bei alten, insbesondere bei gebrechlichen Patienten eine große Rolle, da sie sehr wahrscheinlich gehäuft zu Sturzereignissen mit sekundärer Morbidität und Mortalität führen. Umdrehen ist eine wesentlich komplexere Bewegung als Geradeausgehen. Erste Daten deuten darauf hin, dass IPS-Patienten eine geringere Variabilität als gesunde Personen bei der Durchführung derartiger Drehbewegung aufweisen, und auch Geschwindigkeits- und Beschleunigungsparameter innerhalb dieser Bewegung verändert sind.

\section{Transfers}

Mithilfe von tragbaren Sensorsystemen konnte gezeigt werden, dass IPS-Patienten im Vergleich zu Kontrollen unter Alltagssituationen einen veränderten Bewegungsablauf bei Sitz/Stand- und Stand/Sitz-Transitionen aufweisen. Eine Einschränkung dieser Bewegungen ist, vergleichbar zu Drehbewegungen, verstärkt mit Stürzen assoziiert. Interessant scheinen in dem Zusammenhang vor allem Flexions- und Extensionsphase von Aufstehen und Hinsetzen zu sein. Es gibt Hinweise, dass beim IPS die Variabilität der Parameter eingeschränkt ist, was auf eine verminderte Dynamik der Bewegung und eine verminderte Anpassungsfähigkeit an die äußeren Umstände hinweist.

Aus diesen Beobachtungen lässt sich ableiten, dass durch die Auswertung von Daten, welche durch derartige Sensorsysteme und Algorithmen zur Verfügung stehen, völlig neue Aspekte des IPS zugänglich werden. Es ist z.B. vorstellbar, dass aufgrund derartiger spezifischer Auswertungen gerade alltagsrelevante Defizite mit großer Genauigkeit festgestellt und dann gezielt therapiert werden können.

\section{Herausforderungen der neuen Entwicklung}

Neben den großen Chancen, die diese Entwicklung bietet, ergeben sich auch neue Herausforderungen sowohl für den Patienten, der solche Systeme benutzt, als auch für den Arzt. Zum Beispiel stellt die Validierung der Daten ein enormes Problem dar. Validierung im engeren Sinne bedeutet sicherzustellen, dass ein System auch wirklich misst, was es intendiert zu messen. Dies ist im Labor relativ sicher zu testen. Ein übliches Verfahren ist dabei die Verwendung von 
Sensorsystemen und der simultanen Videoaufzeichnung der Bewegungen, oder aber der Vergleich von Sensor-basierten Daten mit komplexen Labor-basierten Aufzeichnungssystemen. Erste Untersuchungen zeigen allerdings, dass Patienten an sich gleiche Bewegungsabläufe unter Laborbedingungen anders ausführen als in Alltagssituationen ${ }^{9}$. Damit dürfen im Labor gewonnene Erkenntnisse und entwickelte Algorithmen nicht unkritisch im Alltag eingesetzt werden, da damit gerechnet werden muss, dass Spezifität und Sensitivität für die Erkennung von bestimmten Bewegungsabläufen, Symptomen und Defiziten in dem Rahmen nicht so hoch ist wie unter Laborbedingungen. Studien zur Erhebung des medizinischen Nutzens derartiger Technik für die Patienten sowohl beim Arztbesuch, als auch in der häuslichen Umgebung, liegen unseres Wissens derzeit noch nicht vor.

Eine weitere große Herausforderung stellt die Sicherung der Privatsphäre dar. Durch die Erhebung von Daten im häuslichen Umfeld werden ethische und juristische Fragen in einer neuen Dimension aufgeworfen, da insbesondere eine Vielzahl von derartigen Systemen auch mit der Option arbeiten, Daten über das Internet an den Kliniker oder Wissenschaftler zu übertragen. Eine propagierte und Erfolg versprechende Lösung dieses ethischen und datenschutzrechtlichen Problems könnte die Patienten-gesteuerte Krankenakte sein. Dabei ist der Patient der Besitzer seiner elektronischen Krankheits- und Gesundheitsdaten, die er selbstbestimmt entweder vollumfänglich oder auch nur teilweise den verschiedenen Partnern im Gesundheitssystem zur Verfügung stellen und dies auch jederzeit widerrufen kann.

Eine andere Herausforderung stellt die Nutzung derartiger Systeme über längere Zeiträume, evtl. Jahre bzw. über die gesamte Dauer der Erkrankung dar. Mit anderen Worten: Wie können wir erreichen, dass Patienten auch langfristig derartige Systeme benutzen, da auch nur dadurch adäquate und Therapie-relevante Entscheidungen über einen längeren Verlauf getroffen werden können? Dies ist insofern nicht selbstverständlich, als nach aktuellen Studien ${ }^{10}$ ein Drittel der Personen, welche tragbare Sensoren bzw. Apps im Fitnessbereich nutzen, diese nur die ersten sechs Monate verwenden. Es ist vorstellbar, dass durch Nutzung derartiger Sensorsysteme unter Einbindung des Patienten eine hohe intrinsische Motivation beim Patienten erzeugt werden kann, „an der Therapie der Krankheit selbst mitzuarbeiten“. Erste Ergebnisse dazu sind ermutigend: So traten in einer Beobachtungsstudie über 12 Wochen, in welcher IPS-Patienten ein relativ komplexes Sensorsystem zuhause rund um die Uhr verwendeten und dabei Rückmeldung zu Krankheitsaspekten bekommen haben, keine Drop-outs auf ${ }^{11}$. 
Tragbare Sensorsysteme haben durchaus das Potenzial, die Aufnahmeuntersuchung und auch die klinischen Visiten im Krankenhaus, sowie auch die Untersuchung des niedergelassenen Arztes substanziell zu beeinflussen und zu verändern. So können in der klinischen Routine oft durchgeführte Untersuchungen wie standardisierte Gangtests und Transfer (z.B. mittels des Timed-up-and-go-Tests oder der Short Physical Performance Batterie) und Feinmotorik-Testungen nun mit zusätzlich getragenen Sensoren durchgeführt werden. Damit ist kein relevanter zeitlicher Mehraufwand verbunden, und es kann eine Vielzahl von zusätzlichem Datenmaterial generiert werden. Es ist wahrscheinlich, dass mit derartigen Systemen Informationen gesammelt werden können, die dem klinischen Auge entgehen. Beispiele sind Gangvariabilität, Aufsetzwinkel des Fußes beim Gehen und Zeit, in welcher beide Füße beim Gehen auf dem Boden sind (double limb support). Vorhandene Systeme können diese Gangparameter bereits problemlos extrahieren, jedoch stellt die große Bandbreite der physiologischen Gangmuster und die Individualität der jeweiligen Patienten eine große Herausforderung an die Auswertealgorithmen und die medizinische Interpretation der gewonnenen Ergebnisse dar. Hier können individuelle longitudinale Daten, die von (jetzt gesunden Personen, z.B. im Rahmen von Fitness und Sport) gesammelt werden und in der Zukunft für einen individuellen „historischen“ Vergleich herangezogen werden, eine Möglichkeit darstellen, das Problem elegant zu lösen.

\section{Zusammenfassung}

Die Entwicklung von kleinen, tragbaren und auch immer günstiger werdenden Sensorsystemen im Gesundheits- und Fitnessbereich als auch in "Alltags“-Geräten wie dem Smartphone, kann dazu genützt werden, die Qualität der Erfassung von Symptomen insbesondere bei chronischen Erkrankungen deutlich zu verbessern. Das IPS eignet sich aufgrund der Symptomkonstellation und dem chronisch progredienten Krankheitsverlauf besonders für die Etablierung und Anwendung solcher Sensorsysteme. Erste Ansätze zur erfolgreichen Symptomerfassung im häuslichen Umfeld bestehen bereits. Es gibt eine Reihe von Herausforderungen, welche in Zusammenhang mit dieser Entwicklung bewältigt werden müssen. Dabei stehen Validierungsaspekte, Patientenmotivation und Datensicherheit im Vordergrund. Selbstverständlich muss die Relevanz der gewonnen Daten hinsichtlich ihres diagnostischen und therapeutischen Wertes kritisch evaluiert und validiert werden. 
1. van Uem J, Marinus J, Canning C, et al. Health-related quality of life in patients with Parkinson's disease - a systematic review based on the ICF model. Neurosci Biobehav Rev. 2015; Epub ahead of print, PMID:26645499

2. Pasluosta CF, Gassner H, Winkler J, Klucken J, Eskofier BM. An Emerging Era in the Management of Parkinson's Disease: Wearable Technologies and the Internet of Things. IEEE J Biomed Heal informatics. 2015;19(6):1873-1881.

3. Maetzler W, Rochester L. Body-worn sensors-the brave new world of clinical measurement? Mov Disord. 2015;30(9):1203-1205.

4. Maetzler W, Domingos J, Srulijes K, Ferreira JJ, Bloem BR. Quantitative wearable sensors for objective assessment of Parkinson's disease. Mov Disord. 2013;28(12):1628-1637.

5. Müller T, Harati A. Diadochokinetic movements differ between patients with Parkinson's disease and controls. J Neural Transm. 2010;117(2):189-195.

6. Griffiths RI, Kotschet K, Arfon S, et al. Automated assessment of bradykinesia and dyskinesia in Parkinson's disease. J Parkinsons Dis. 2012;2(1):47-55.

7. Klucken J, Barth J, Kugler P, et al. Unbiased and mobile gait analysis detects motor impairment in Parkinson's disease. PLoS One. 2013;8(2):e56956.

8. Mancini M, El-Gohary M, Pearson S, et al. Continuous monitoring of turning in Parkinson's disease: Rehabilitation potential. NeuroRehabilitation. 2015;37(1):3-10.

9. Hammerla N, Fisher J, Andras P, Rochester L, Walker R, Ploetz T. PD Disease State Assessment in Naturalistic Environments using Deep Learning. Austin, Texas; 2015. p. AAAI - 2015.

10. Ledger D. Wearables Abandonment rates are not improving. Endeav Partners. 2015;

11. Ferreira JJ, Godinho C, Santos AT, et al. Quantitative home-based assessment of Parkinson's symptoms: the SENSE-PARK feasibility and usability study. BMC Neurol. 2015;15:89. 